\title{
MORTALIDAD INFANTIL CHILENA
}

\author{
Dr, GULLERMO ADRIASOLA
}

Cátedra de Higiene Macerno-Infantil, Escuela de Salubridad, Universidad de Chi.e

La mortalidad infantil ha sido una de las vías de drenaje de la energía humana más persistentes a través de la historia. La falta de estadísticas impide comparar, en este aspecto, otras épocas con los tiempos modernos. Pero basta recordar cómo fueron diezmadas nuchas descendencias reales, datos que conocemos porque fueron registrados por el interés en la sucesión al trono, para comprender que si éste era el riesgo infantil en ese medio social, el de la generalidad de la población, campesinos, y esclavos, debió ser tremendo. El estudio de la osificación de los esqueletos humanos precristianos $y$ de las inscripciones de edad en las tumbas, ha hecho estimar que la expectación de vida griega y egipcia debió estar entre 20 y 30 años de edad (1). Concomitantemente, la mortalidad infantil tuvo que ser muy elevada. Desde entonces hasta el Renacimiento, la expectativa de la vida parece que se mantuvo igual. En Londres, alrededor del año 1550, sólo la mitad de los nacidos vivos llegaba a la edad de 8 años (2). Desde entonces, Inglaterra no ha cesado de mejorar sus tasas. Mientras tanto, en Chile, a comienzos de este siglo, $1 \mid 3$ de los nacidos moría antes del año de edad, situación similar a la de Londres de hace 4 siglos.

En China, hasta no hace mucho, no se consideraba contable un niño mientras no hubiera tenido viruela, dado el alto riesgo de contagio temprano y de muerte.

La mortalidad infantil está registrada muy irregularmente en el mundo, tanto en cuanto a acuciosidad como en cuanto al tamaño de sus tasas. Hay paúses con población indígena dispersa que escapa a todo registro o es eliminada de él. En Nueva Zelandia la población maorf, que es del $6 \%$ del total, no es incluída en las tasas oficiales, las segundas más bajas del mundo. Algunas estadísticas hispano-americanas que aparecen demasiado bajas en relación con los países de similares condiciones sanitarias, económicas y culturales, podrían adolecer del mismo error. (Ver nota de tabla $N^{\circ} 1$ ).

En Chile se viene haciendo censos más o menos cada diez años, desde hace más de un siglo. El regístro de nacimientos y muertes se hace con bastante propiedad, desde 1875 . A lo menos, nuestras tasas crudas son dignas de confianza. Passmore, de Edimburgo, dijo aquí, con gran propiedad: "Chile es el país con las mejores malas tasas del mundo" (3).

La mortalidad infantil a comienzos de sigla era de 152 en Inglaterra, de 150 en U. S. A. y de 134 en Suecia. En Chile, en que la mortalidad infantil era de 340 , recién hoy hemos alcanzado esos valores.

TABLA N 1

Orden de las 8 más altas y de las 8 más bajas tasas registradas entre 101 países $y$ territorios, por las N.U. (1949) *

Pais o territorio

Calidad de registro

Tasa

Suecia

Islandia

Nueva Zelandia (sin maories)

Austialia

Hawai

Holanda

Noruega

Estados Unidos

Costa de Oro

Bolivia

Borneo

Barlados

Colombia

Egipto

Aden

Chile

\begin{tabular}{l}
$\mathrm{C}$ \\
$\mathrm{C}$ \\
$\mathrm{C}$ \\
$\mathrm{C}$ \\
\hline \\
\hline
\end{tabular}

23,3

28.7

28.8

25,2

25,3

26,8

27,7

31,3

124,8

127,2

128,3

192,6

134,1

] 35,5

158,6

169,0

- Anuatio demografico 1952. De los 101 paises y territorios, so.o 45, inclutdo Chile, aparecen con registros completos o virtualmente completos (C), mientras 24 aparecen con registros incompletos (I) y Ios restantes. sin indicación. De los 8 países con alta mortalidad hay varios en que, según informaciones autorizadas, la mortalidad infantil real es mas del doble de la registrada. 
Desde entonces a esta parte, esos países, como casi todos los países o ciudades, registran un importante descenso de la mortalidad infantil, la que llegó a reducirse a la $5^{\mathrm{a}}$ o $6^{\mathrm{a}}$ parte.

En Chile, el proceso ha sido notoriamente menor a pesar de su importancia. Comienza a manifestarse con claridad desde 1934 . Desde 1900 ha bajado el $39 \%$ o sea a la cifra actual de 134\%; su descenso es paralelo al descenso de la mortalidad general en los primeros años de este siglo, e irregular como ella, sugiriendo la influencia de las enfermedades infecciosas (4). EI descenso brusco de 1928 es debido a cambios en el registro de nacidos vivos. (Gráfico 1).

Es imposible determinar cuál es la participación que le corresponde en esa mejora de las tasas chilenas, al progreso social-económico, o a factores que han sido propuestos como casuales; la inauguración de la pediatría moderna en el $2^{\circ}$ decenio, la acción de instituciones médico-asistenciales como el S. O. O. y, en el momento preserite, de la salubridad moderna.

Llama sí la atención que mientras en países muy evolucionados la baja de la mortalidad infantil afecta preferentemente al mayor de un mes, en nuestro país sucede lo contrario. Así, en Suecia y en los Estados Unidos la mortalidad neonatal excede en $200 \%$ y en un $100 \%$ respectivamente, a la infantil tardía, mientras en Chile, ésta excede a la neonatal en un $100 \%$.

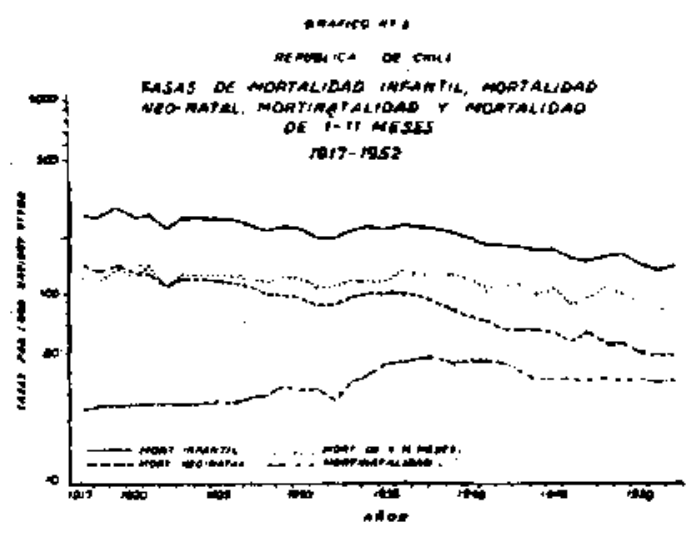

O sea, en Chile como en atros países no desarrollados, la probabilidad de morir del lectante es mayor después del mes de edad que antes y mayor aún, si se le compara con los países líderes. El ambiente de esos países sub-desarrollados es más hostil que en estos otros, para el lactante mayor de 1 mes. La eliminación de gran parte de los riesgos ambientales en los países líderes destaca la mortalidad por causas natales y prenatales, más difíciles de influir.

Romero y Ugarte (4) y Moroder ya habían sugerido la posibilidad de que esta diferente relación de mortalidad neonatal e infantil tardía, fuera consecuencia de la diversidad de las etapas históricas comparadas.

Gracias a un retraso económico y sanitario desacorde con el proceso biodemográfico, nuestros paises estarían patentizando etapas que los paises líderes vivieron antes de la preocupación bioestadística.

El estudio de Moroder y cols. (5), aporta antecedentes en favor de tal aguda crisis. Ellos afirman que habría tres etapas históricas. En la primera, cuando la mortalidad infantil es muy alta, la mortalidad neonatal es mayor que la infantil tardía. La mayoría de los países habrían pasado esta etapa antes de que hubiera registros estadísticos adecuados. En Chile, esta etapa habría terminado al final del $2^{\circ}$ decenio de este siglo, prolongándose hasta 1936, en el medio rural, cuando la curva de la mortalidad neonatal bajó cruzando la de la mortalidad infantil tardía. (Gráfico 2).

$$
\text { GAARIO NOZ }
$$

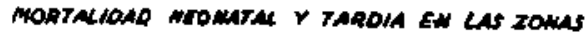

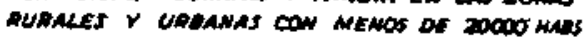

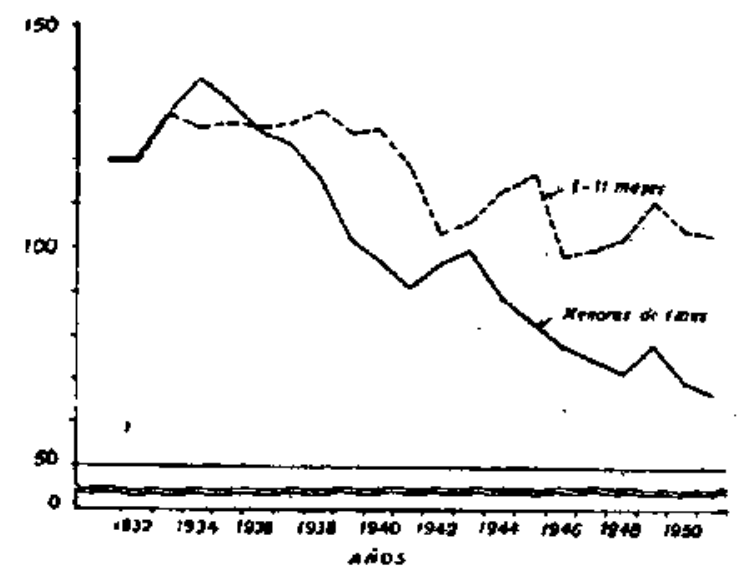

El segundo período es cuando se registra un descenso de las tasas y cuando la mortalidad infantil tardía supera a la neonatal. Esta etapa termina cuando la mortalidad tardía baja cruzando la curva de la morta- 
lidad neonatal. Nueva Zelandia, EE. UU., Suecia la abandonaron antes de 1930. Chile se encuentra, aún, en esta etapa.

Efectivamente, en Chile, la mortalidad neonatal ha descendido en un $45 \%$, durante los últimos 15 años, hasta 1952 tanto en las ciudades mayores de 20.000 habitantes como el resto del país. Entretanto, el descenso de la mortalidad infantil tardía en las comunidades de menos de 20.000 habitantes no alcanza al $20 \%$, manténiéndose aún en la elevada tasa actual de 94 o/oo. En las ciudades con más de 20.000 habitantes descendió un $\mathbf{5 0 \%}$, siendo la tasa actual de $85 \mathrm{o} / 00$.

Antes de 1944 la mortalidad tardía era mayor en las ciudades de 20.000 habitantes 0 más, que en el resto del país. Desde 1947, para adelante, la mortalidad tardía se hace menor en las ciudades que en el resto del país.

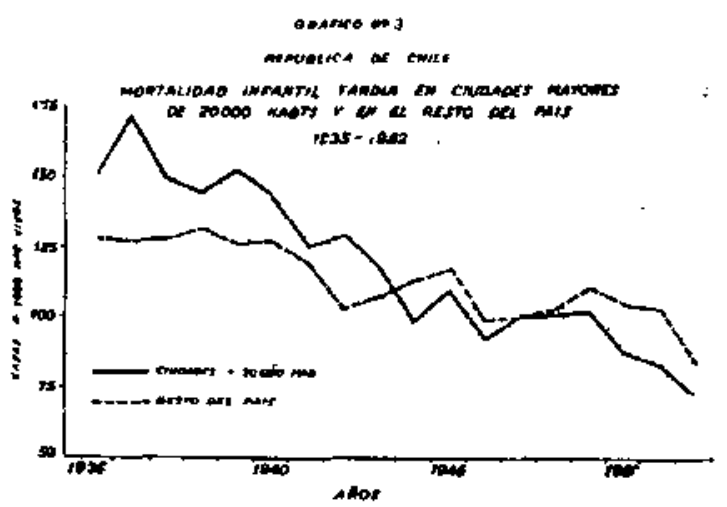

Ciertos o no estos interesantes alcances a la tendencia histórica de la mortalidad infan- til, es el hecho que la comparación de los paises con las más altas tasas de mortalidad infantil y de las más bajas, revela que estos últimos están perdiendo alrededor de 120 vidas menores de un año por mil nacidos vivos, en comparación con los otros. Vidas que estos países ahorran y que nos señalan lo prevenible que nosotros no pudimos prevenir.

¿Cómo y por qué mueren estos niños? Por nuestro mejor conocimiento de las tasas chilenas y porque el método epidemiológico que seguiremos es válido para el análisis de cualquier comunidad nos referiremos al caso nacional.

Sexo.-En Chile nacieron $51 \%$ de hombres contra $49 \%$ de mujeres durante el quinquenio 1947-1951. Tanto la mortalidad neonatal, como la infantil se compone de un mayor porcentaje de hombres que de mujeres en el orden de 54 y $46 \%$ respectivamente, diferencias estadísticamente significativas. Este hecho que es común a todos los países, y que afecta igualmente a la mortinatalidad, podría estar relacionado con caracteres diferentes en cada sexo: genéticos, fisiológicos o de crecimiento y desarrollo.

Edad.-La población de menores de un año es, en Chile, algo superior al $3 \%$ de la población total. Sin embargo, la mortalidad infantil hace el tercio del total. Del mismo modo, la mortalidad neonatal es más de un tercio de la infantil, en circunstancias que se refiere a un período 12 veces menor. La mortalidad de la primera semana es más de la mitad de la neonatal y la del primer dia, es más de la mitad de la primera semana.

\begin{tabular}{|c|c|c|c|c|c|c|}
\hline Edad & \multicolumn{3}{|c|}{ Número } & \multicolumn{3}{|c|}{ Porcentaje } \\
\hline Primer día ....................... & 3.868 & & & 3,77 & & \\
\hline 1 a 7 dias & 2.915 & & & 2,81 & & \\
\hline 8 a 12 dias & $\mathrm{J} .818$ & & & 1,76 & & \\
\hline 16 a 23 dias ................. & 1.744 & & & 1,69 & & \\
\hline 24 a 30 dias ............... & 1.449 & & & $\mathrm{I}, \mathbf{4 6}$ & & \\
\hline Primer mes & 11.814 & 11.814 & & $1 I, 43$ & 11.48 & \\
\hline Segutrdo mes ………..... & & 4.016 & & & $\mathbf{3}, 89$ & \\
\hline Tercer mes & & $\mathbf{3 , 0 7 0}$ & & & 2,97 & \\
\hline Primer trimestre & & 19.900 & 19.900 & & 18.29 & 18,20 \\
\hline Segundo trimestre & & & 6.450 & & & 6,65 \\
\hline Tercer trimestre .................. & & & 3.715 & & & 3,59 \\
\hline Cuarto trimestre & & & 2.999 & & & 2,90 \\
\hline Total & & & 32,064 & & & 31.01 \\
\hline
\end{tabular}

Estos hechos son similares en todos los países y reflejan la mayor dificultad del niño menor para adaptarse a las nuevas condicio- nes ambientales extrauterinas y a la excepcional tensión que soporta durante el parto.

El estudio de Cabello (8) en la Unidad Sa- 
nitaria Quinta Normal (Santiago, Chile) demuestra que la znortalidad neonatal es mayor en hijos de mujeres muy jóvenes o mayores de 40 años; mayor en primogénitos y después del $4^{\circ}$ hijo, coincidiendo con las conclusiones de Yerushalmy (9) .

Madurez y peso.--La mortalidad infantil está estrechamente ligada a la madurez y peso del niño al nacer, elementos ambos que que se confunden para todo proceso estadístico y práctico. Las tablas de vida que calculamos para los recién nacidos en algunas maternidades de Santiago en 1950 (6) nos demostraron que la mortalidad del prematuro es 18 veces mayor que la del recién nacido maduro, en la primera semana de vida.

Del mismo modo comprobamos que el prematuro muere en mayor proporción a medida que su peso de nacimiento baja de los 2.500 gramos.

En el Manual del Children's Bureau (6) se consignan las estadísticas de 4 hospitales americanos con basta experiencia en cuidado de prematuros y los cuales alcanzaron en 1945, grandes progresos en la sobrevida de estos seres. Los prematuros con peso de nacimiento entre 2.001 y 2.500 grs. sobreviven en el 92,8 y $95,8 \%$. Los de peso entre 1.501 y 2000 grs., sabreviven en el 50 y $58,5 \%$. En Estados Unidos, de los prematuros que mueren antes de cumplir el primer año de edad y son registrados, mós del $50 \%$ mueren en el primer día.

Raza.-Las escasas informaciones hispanoamericanas que conocemos nos dicen que el niño indígena muere más que el blanco. En Estados Unidos, la mortalidad infantil de negros, amarillos e indígenas era, en 1947, de 48,5 mientras la de los blancos era de $30.1 \%$. En Algeria la mortalidad de los musulmanes es mavor que la de los europeos: en Nueva Zelandia. los blancos tienen tasas de 23 mientras la de los maoríes era de 70 en 1949 , etc.

No sabemos aún qué influencia tiene el factor racial en la mortalidad infantil, pero estamos seguros, sí, que las diferencias senaladas se asocian a muchas e importantes variables, cuya influencia es mayor en el factor racial. Nos referimos a cultura, alimentación, vivienda, saneamiento, etc.

Factores económico-sociales. En general, la literatura ofrece bastantes evidencias de que el bajo nivel económico-social provoca mayor morbilidad $\mathrm{y}$ mayor mortalidad.

Pero en ningún grupo humano es tan es- trecha esta relación causal, como en el infantil. Se ha dicho, con razón, que la mortalidad infantil describe el progreso económicosocial y sanitario de un pueblo.

Levine y Crocco (10) relacionaron la baja del poder adquisitivo de los salarios chilenos, con el alza de la mortalidad infantil en los años siguientes a 1930 .

Efectivamente, se observa que el alza de Ia mortalidad infantil en 1931 a 1934, coincide con la caída del poder adquisitivo de la moneda, alcanzando ambas tendencias su punto extremo en 1934. (Gráfico 4).

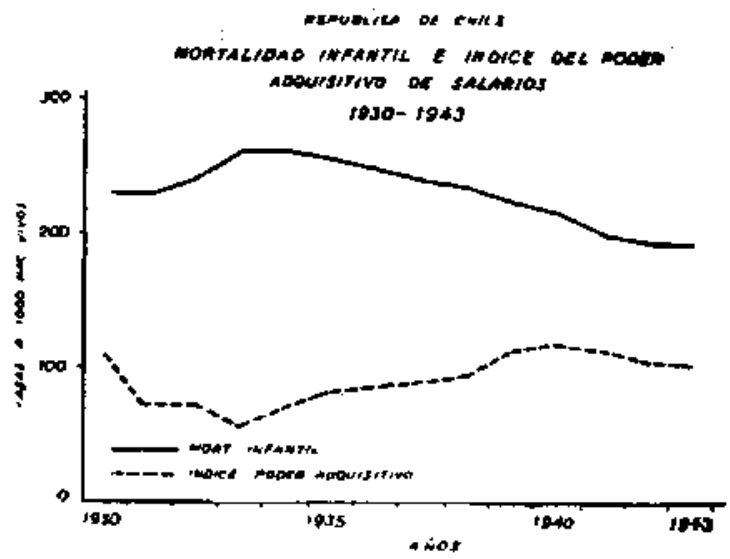

Si los factares adversos son las causas del alza de la mortalidad infantil después de 1932, deberíamos suponer que alguna influencia tienen en el alza coetánea de la mortinatalidad o de la mortalidad maternal por abortos. Después de observar la asociación de tales curvas (Gráficos 1 y 2 de Hig. Maternal y 4 de Hig. Infantil), estaríamos en condiciones de decir que la crisis económica de Chile, iniciada en 1930, aumentó la mortalidad fetal e infantil, al mismo tiempo.

Sin embargo, a partir de 1940, el continuado descenso de la mortalidad infantil no guarda relación con el poder adquisitivo de los salarios. Es evidente, pues, que si este factor tiene influencia existen otros cuyo peso puede ser igualmente importante.

Influencia de la concentración de habitantes.-La mortalidad infantil en Chile, durante 1952, fué de 109 o/oo en las ciudades de 20.000 o más habitantes y de $155 \mathrm{o} / 00$ en el resto del país, y la mortalidad neonatal fué de $36 \% / 00$ y de $61 \% / 00$, respectivamente.

El Gráfico $\mathrm{N}^{0} 5$ demuestra que existe una asociación positiva entre ruralidad y mortalidad infantil. 
GRATHEO wS

nePUELICA DE cHice

CORRELACION ENTHE MOATALIOAO WFANTHC $Y$ POACENTAJE DE AOBLACION UREAMA AOA PAOVINCIAS_ 1OSJ

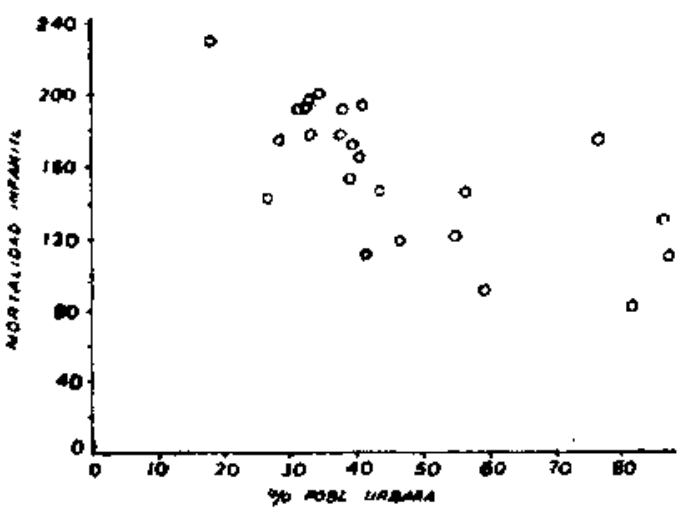

La experiencia americana demuestra que el saneamiento y demás ventajas urbanas, dominaron los mayores riestos iniciales del hacinamiento urbano, hace algunos decenios, hasta superar las bajas tasas de la mortalidad infantil derivadas de Ia vida agreste. Parece, aún, que las pequeñas ciudades de un par de miles de habitartes tienen mayor mortalidad infantil que las ciudades más grandes $y$ que las zonas rurales, como si habiendo perdido las ventajas del campo, aún no gozarán de las ventajas urbanas.

La urbanización incluye una cantidad grande de variables. No sabemos cuál es el, o cuáles son, los factores determinantes o más influventes de la disminución de la mortalidad infantil en las ciudades. En otros, por ejemplo, la mortalidad infantil también está asociada a la hospitalización de las parturientas. Altenderfer y Crowther (11) estudiaron la mortalidad infantil, observando una asociación negativa con el tamaño de la población en áreas urbanas, con el porcentaje de regros, con los ingresos "per cápita" y con los nacimientos en hospital. Observaron también que tales asociaciones negativas desaparecen con el método de standardización y de correlación parcial; salvo la entre mortalidad infantil y proporción de nacimientos en hospitales, que es independiente de los otros 3 factores. En otras palabras, la asociación de mortalidad infantil baja con ingresos "per cápita" altos, o con alta proporción de blancos, es una consecuencia de la asociación de estos factores, con porcentajes altos de nacimientos en hospital.
La correlación entre mortalidad infantil y porcentaje de nacimientos en hospital no indica necesariamente relación causal. No es la simple hospitalización, sino que son todos los cuidados y facilidades que ello invoJucra, los que salvan muchas vidas. La hospitalización, con su cortejo de atenciones matemo-infantiles de alta calidad, parece importante en la prevención de la mortalidad infantil en U.S.A.

El Gráfico No 6 demuestra que en Chile hay, también, una correlación inversa entre el porcentaje de partos ocurridos en hospital y la mortalidad neonatal por provincias. El cálculo demuestra que la correlación es estadísticamente sirnificativa.
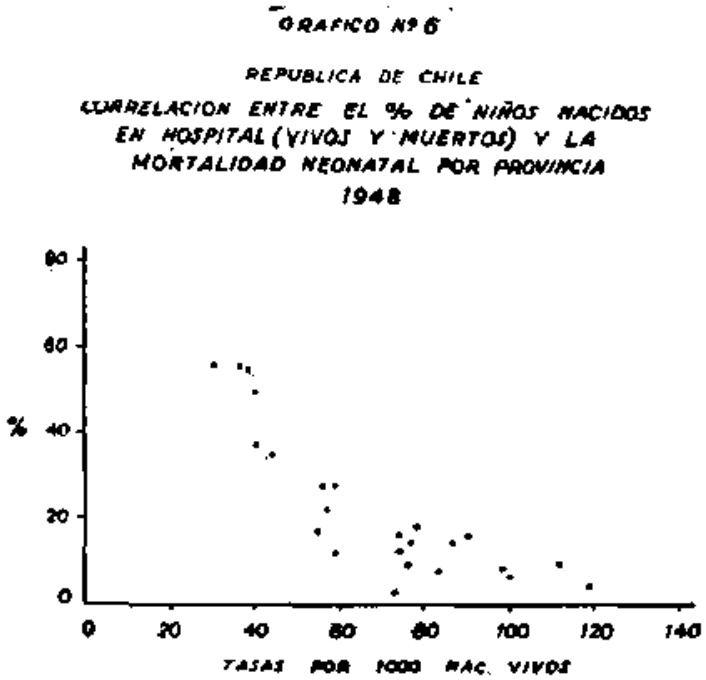

Legitimidad.-En Chile, según las últimas estadísticas, la proporción de niños menores de un año, ilegítimos, es de $19 \%$ al nacer, y es de más del $30 \%$ al morir. El ilegítimo tiene, pues, una probabilidad notablemente mayor de morir durante el primer año. Debemos puntualizar que la ilegitimidad está, también, fuertemente asociada a la prematuridad, a la juventud extrema de la madre y a gran cantidad de variables; emocionales, económicas y sociales.

Alimentación.-Los clásicos estudios de B. Burke (15 Hig. Mat.) y de Riquelme (9 Hig. Mat.) y otras investigaciones demuestran que la adecuada alimentación de la embarazada garantiza la sobrevida del niño.

La literatura está llena de cifras y afirmaciones que prueban que la alimentación natural del lactante significa morbilidad $y$ mortalidad infantil menores que las registradas para el lactante a biberón. La alimen- 
tación artificial está también asociada a variables emocionales, económicas y sociales. En los países anglosajones se ha conseguido las bajas tasas actuales de mortalidad infantil, con el concomitante mayor porcentaje de alimentación artificial, conocido en la historia. Mas, no sabemos si estas bajas tasas hubieran sido mayores o no, si se hubiera alimentado naturalmente a los lactantes, manteniendo iguales las otras variables.

S. Stevenson, siguiendo todos los requerimientos estadísticos, investigó dos grupos comparables de lactantes, unos alimentados a biberón; el otro, alimentado naturalmente (12). Concluyó que, la única diferencia entre uno y otro grupo parecería ser una incidencia de $1,43 \%$ de infecciones respiratorias durante el $2^{\circ}$ semestre de la vida, en los alimentados artificialmente; contra $0,95 \%$ en los otros. "Cuando la alimentación artificial es Ilevada a cabo propiamente en un grupo de madres seleccionadas $y$ cuidadosamente vigiladas, se compara favorablemente con la natural."

Pero estas condiciones experimentales no existen en la práctica, para los hijos de madres no seleccionadas del mundo; quienes son la abrumadora mayoria. La alimentación artificial es un riesgo tramendo de infecciones, aparte de emocional.

La anécdota que el mismo $\mathbf{S}$. Stevenson cuenta (10) es muy ilustrativa: Durante la guerra pasada los americanos que regentaban un hospital militar en el interior de Egipto comprobaron que gran parte de las madres no podían amamantar a sus hijos a causa de estar agotadas por la malaria. Es de notar que, en cambio, los lactantes habían escapado al contagio, probablemente por la arraigada costumbre de envolverlos y cubrirlos, casi totalmente. con Jas ropas.

Existiendo la imposibilidad de disponer de leche de vaca para suplementar la alimentación natural, recurrieron a la leche dromedaria; ensayando en el propio hospital una fórmula con ésta. El éxito fué rotundo. Los lactantes toleraron la nueva fórmula v progresaren bien. Entónces los americanos trataron de difundir en la población civil la nueva técnica. El resultado fué desastrosn. En manos de los nativos de bajo nivel cultural, la fórmula a base de leche de dromedaria se convirtió en el infanticidio organizado de los beneficiarios.

Mardones (13) relaciona Ia mortalidad in- fantil con la cantidad de leche repartida por los Centros Infantiles en diversas localidades de Chile. La correlación negativa parece altamente significativa. Debemos puntualizar, una vez más, que junto a la acción de la leche repartida, deben jugar gran cantidad de variables (de asistencia pediátrica y otras), como para que no estemos autorizados a atribuir determinado carácter a dicha asociación.

Infiuencia de ta natalidad,-La mortalidad infantil y la natalidad han estado asociadas en todas partes y todos los tiempos.

En Chile, la mortalidad infantil bajó desde 1932 a 1951 en un $22,5 \%$, mientras la natalidad descendió en un $4,7 \%$ Las ciudades mayores de 20.000 habitantes, que ofrecen un buen registro de nacimientos y muertes, muestran una correlación significativa entre ambas tasas. (Gráfico No 7 ).

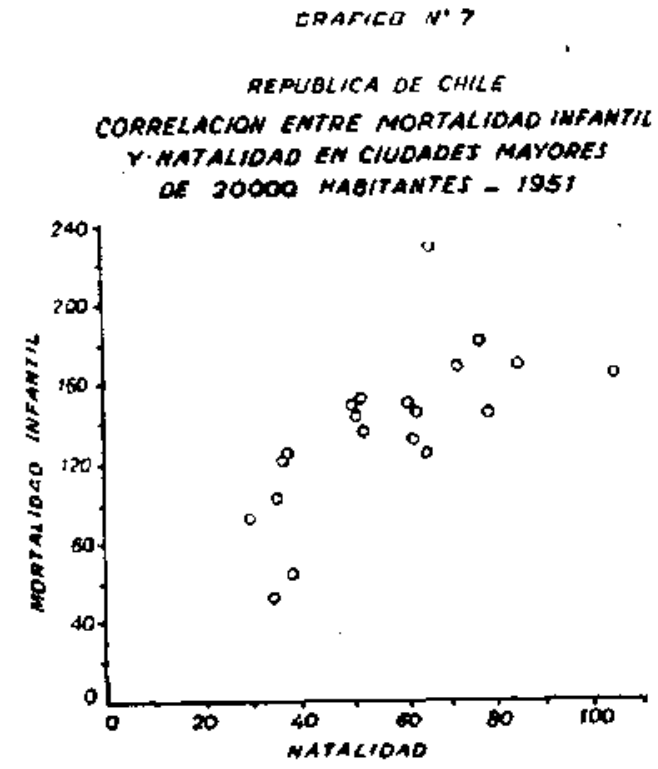

Los países con las tasas más bajas de mortalidad infantil tienen natalidad alrededor de 20 o tasas de natalidad inferiores a $20 \mathrm{o} / \mathrm{oo}$, tienen una mortalidad infantil inferior a 58 o/ oo y 4 de ellos, tasas inferiores a $35 \% / 00$.

Los países con tasas altas, tienen generalmente registros incompletos lo que hace inútil establecer correlaciones matemáticas, en ellos.

Nicolai, en 1933, analizó está correlación. usando las tasas de la Liga de las Naciones. Concluye que ambas, mortalidad infantil y natalidad, están asociadas positivamente (14). 
Tal asociación está influída, necesariamente por las variables económicas y sociales. Las tasas más bajas de natalidad y mortalidad infantil se observan principalmente entre los pequeños países neutrales: Suecia, Noruega, Holanda, Dinamarca, Suiza, Luxemburgo y Mónaco, con las opuestas excepciones de Inglaterra y Portugal.

Parece que, en general, la baja de la mortalidad infantil ha precedido a la natalidad. Así ocurrió en Chile. Es probable, en todo caso, que la prolongación de la lactancia natural y la conservación de un número mavor de hijos vivos haya influído tanto en la Roma y Grecia antigua, como en la época moderna, en la tendencia a evitar nuevos embarazos.

Influencia de las estaciones.-La mortalidad infantil presenta en Chile una definida variación estacional con alza estival y el punto de más declive en invierno. (Gráfico 8).

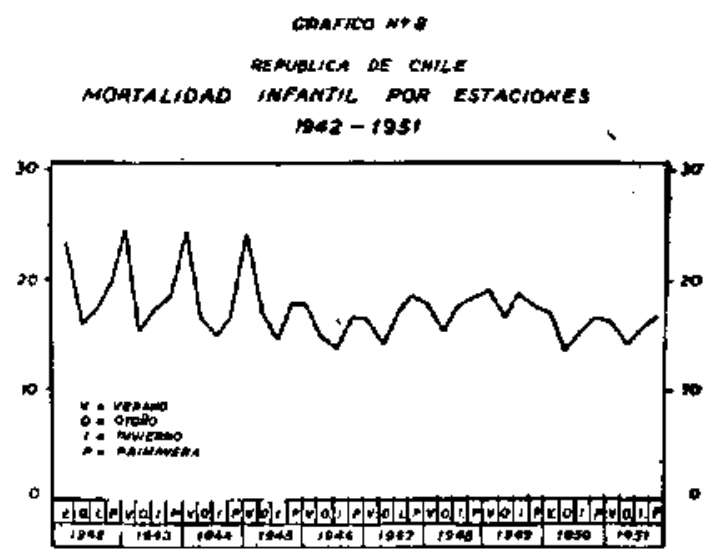

Hace excepción a esta distribución la mortalidad de la zona Austral en donde la mayor mortalidad infantil aparece en el invierno. En esta zona la baja temperatura reinante durante todo el año - media anual de $6^{\circ}$, aproximadamente- y demás condiciones climatéricas extremas, apagan la patología infecciosa digestiva y aumentan la respiratoria.

Dicha variación estacional se viene mocerando desde 1940, en Chile. La curva más alta de la mortalidad estival de las ciudades de más de 20.000 habitantes, definitivamente cruza en 1937 la curva de las poblaciones restantes del pais por debajo de ésta (15). Hace 40 años New York tenía esta misma distribución estacional. La pasteurización de la leche e higienización de los establos, más el saneamiento del agua potable y de Ios sistemas de alcantarillado parecen haber sido decisivos para borrar el alza estival y bajar la mortalidad infantil total. Hoy, en el total de EE. UU., la mortalidad infantil muestra su punto más alto en el invierno y el mayor declive en verano. Del mismo modo, los niños nacidos en verano $\mathrm{y}$ a comienzos Ael otoño tienen mayores probabilidades de cumplir el año de edad que los nacidos durante e] invierno, tal vez debido a que ésos afrontan Ias infecciones crecientes de invierno $v$ primavera cuando han alcanzado mayor edad y adaptabilidad (16). Es cierto aue, en verano. enfrentan el rieseo de las diarreas estivales, pero en países como EE. UU. este riesgo se ha hecho mínimo gracias al saneamiento.

Parere oue nosotros estuviéramos reciên iniciando el proceso citado. Efectivamente. la mortalidad infantil chilena ha bajado deste el trienio 1942-1944 al 1949-1951 en un $17.8 \%$. Pero esta baja se hizo en un $28 \%$ en la mortalidad estival y sblo en un $10 \%$ en los de las otras estaciones del año.

\section{Resumen.}

El estudio epidemiológico precedente demuestra que la mortalidad infantil se afecta por factores genéticos como el sexo del niño, la edad de la madre y la paridad $\mathrm{y}$, aún, en cierto modo, por el peso del niño, sobre los cuales nuestra influencia es costosa $y$ de resultados escasos.

En cambio, el ambiente, que influve pesadamente sobre la mortalidad infantil, aparece más susceptible de ser favorablemente modificado.

Es difícil jerarauizar la influencia de cada una de las variables sociales, sobre la mortalidad infantil. dada su estrecha $\mathrm{y}$ cambiante interacción. Pero, para beneficio de muestras responsabilidades técnicas. la experiencia demuestra que disponemos de algunos elementos de acción que han demostrado su eficacia, en toda clase de comunidades. Nos referimos al Saneamiento e Higiene Infantil, incluyendo, en ésta, las técnicas alimentarias.

Passmore admiró nuestras instituciones dedicadas a la salud pública, pero expresó en su informe: "El visitante extranjero puede, sin embargo, pesar la eficacia de introducir 
este elaborado y costoso esquema en un país, en el cual la mayoría de los habitantes no tienen abastos adecuados de leche, $y$ viven en increíbles condiciones de habitación, sin agua potable adecuada y desprovistos de alcantarillado" (3).

Por ello, es indispensable acentuar y repetir que la Higiene Infantil no puede desconectarse de un plan general de salubridad, especialmente, del saneamiento ambiental.

Causas de muertes.-Las causas de la mortalidad infantil, expresadas por entidad nosológica, cualesquiera que sean los componentes sociales y económicos, tienen la virtud de mostrarnos. los puntos de ruptura del equilibrio huesped-ambiente $y$ orientar nuestra labor sanitaria.

\section{TABLA No 3}

Tasas y porcentajes de la Mortalidad Infantil por causas. Chile, 1949.

\begin{tabular}{|c|c|c|c|}
\hline Cansas & $\mathrm{N}$ & Tasa & $\%$ \\
\hline $\begin{array}{l}\text { Neutmonia, Bronconed. } \\
\text { monia y Grippe } \\
\text { Debilidad conqenita }\end{array}$ & J0.234 & 53,9 & 31,9 \\
\hline prematuridad & 6.646 & 35,0 & 20.7 \\
\hline Diarrea enteritis & 5,410 & 28,5 & 16,9 \\
\hline $\begin{array}{l}\text { Infecciosas y parasita- } \\
\text { rias (menos grippe) }\end{array}$ & 1.759 & 9,3 & 5.5 \\
\hline Distrofia y atrofia & 1.783 & 9,3 & 5,6 \\
\hline Meningitis simple & 1.189 & 6.0 & 35 \\
\hline Asfixia & 633 & $\mathbf{3 , 3}$ & 19 \\
\hline Tralumas parto & 520 & 2.7 & 1,7 \\
\hline Malformación congénita & 242 & 1,2 & 0.8 \\
\hline Intedeterminadas & 1.149 & 6,0 & $\mathbf{3}, \mathbf{5}$ \\
\hline Orims & $2{ }_{2}^{4} 449$ & 15,5 & 7,9 \\
\hline TOTAL & 32,064 & 169.0 & 100,0 \\
\hline
\end{tabular}

\section{TABLA NO 4}

Número, tasas y porcentaje de la mortalidad infantil para las causas principales en EE. UU. 1947, y Suecia, 1591

\section{Causas}

1. Prematuridad

2. Malformaciones congénitas

8. Netmonia todas formas e influen $\begin{array}{lrr}\mathbf{2 a} & 3,6 & 11,18 \\ \text { Tranmas del parto } 3,5 & 10,87\end{array}$

5. Asfixia y atelectosis $1,6 \quad 4,97$

6. Diarreas enteritis 1,5 4, 506

7. Infracriosas

6. Causas violentas Otras

TOTAL
$-$

$63 \quad 1957$

$32,22100--$

$$
323 \quad 2,94 \quad 11,44
$$$$
-\quad-\quad-
$$$$
\text { - }-1-
$$$$
\begin{array}{lll}
67 & 0,61 & 2,37
\end{array}
$$$$
65 \quad 0.59 \quad 2,30
$$$$
\begin{array}{lll}
56 & 0.42 \quad 1.63
\end{array}
$$$$
243 \quad 2,21 \quad 8,57
$$

$2,82324.03$ 100.-
Las tablas 3 y 4 demuestran la diversa distribución de las causas de muerte, entre países saneados y uno por sanear.

Mientras en Suecia y Estados Unidos Ios riesgos prenatales o natales causan el $73 \mathrm{y}$ $67 \%$ del total de las muertes, respectivamente, y las infecciones causan sólo el $16_{\%}^{\%}$, en ambos; en nuestro país, el $54 \%$ de las causas son infecciosas y el $26 \%$ natales y prenatales (1948).

Creemos indispensable puntualizar que nuestra certificación de causas de muerte contiene errores que los países de tradición estadística ya han superado. El voluminoso rubro de muertes por enfermedades respiratorias agudas debe estar infestado de diagnóstico de cuadros terminales.

Sabemos que esos cuadros, cuando no son procesos finales, se comportan como las infecciones respiratorias agudas, en cuanto a distribución estacional. $O$ sea, que aumentan su frecuencia cuando las variaciones o grados de la temperatura, presión atmosférica o humedad son mayores y cuando el hacinamiento bumano acorta la distancia boca a boca que favorece el contagio respiratorio. En suma, que aumentan en invierno y meses próximos.

En efecto, durante 1951, en Chile, los muertos mayores de 1 año por tales cuadros, aumentaron desde la cifra más baja registrada en febrero, a través del otoño a invierno, sostenidamente, hasta alcanzar la cifra más alta en noviembre. (Gráfico $\mathrm{N}^{9}$ 9).

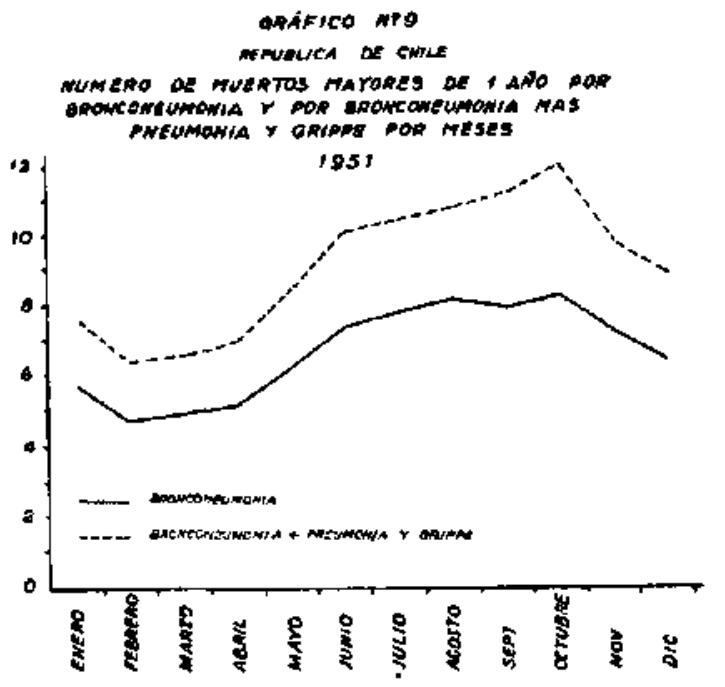

Pero en los menores de 1 año la curva es opuesta. El vértice está especialmente en 
febrero con los valores más bajos entre abril y agosto.

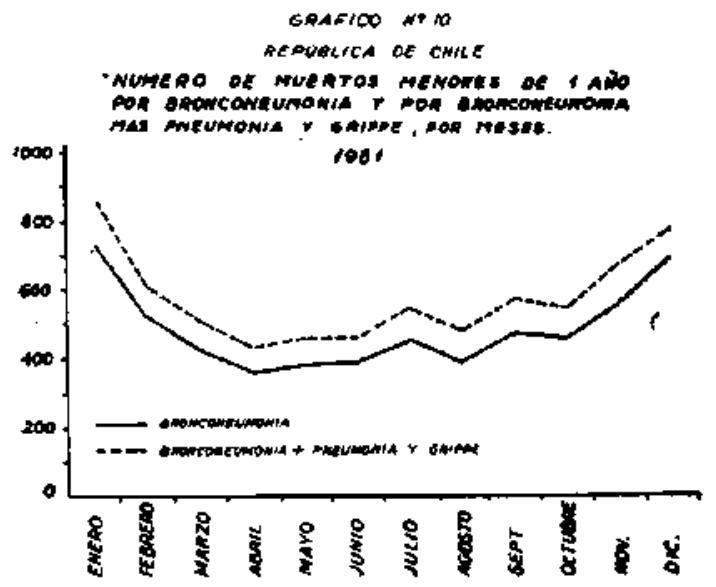

Esta distribución estival es característica de la patología infecciosa digestiva. El Gráfico No 11 expresa dicha oposición.

QAAFICO NTI

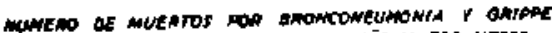

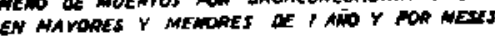
CNLE = IOSI

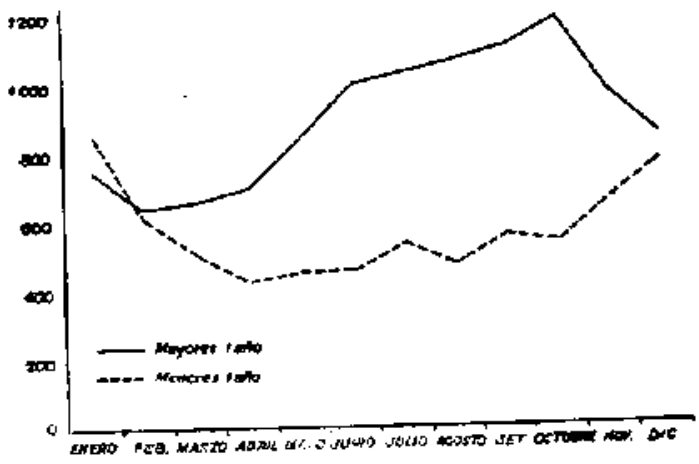

Como la certificación médica de estas muertes no difiere grandemente $(48 \%$ en los mayores de 1 año y 54,07 en los menores); debemos suponer que no juega un rol importante, en el diverso comportamiento de las curvas de ambos grupos. Además, la mortalidad infantil, que tiene una proporción mayor de certificación médica, es la que contradice la experiencia epidemiológica. (La certificación médica de todas las muertes chilenas alcanzó al $66 \%$ en 1952).

Durante 1951, la mortalidad infantil fué el $30,65 \%$ de la mortalidad general y la inortalidad infantil por enfermedades respiratorias agudas fué el $33,59 \%$ de la mortalidad general por enfermedades respiratorias agudas, en los meses comprendidos entre abril y septiembre. $\mathrm{O}$ sea, durante el otoño $e$ invierno. Sin embargo, en primavera y verano, las muertes de menores de 1 año aumentan hasta alcanzar el $43,8 \%$ de la mortalidad general, por estos mismos cuadros.

Tabla No 5

Murtes por infecciones respiratorias agudas, seguin estaciones y grupos de edad. Chile, J95I

\begin{tabular}{|c|c|c|c|c|c|}
\hline Mes & $\begin{array}{c}\text { Mayotes } \\
1 \text { año }\end{array}$ & $\begin{array}{l}\text { Menores } \\
1 \text { año }\end{array}$ & Mes & $\begin{array}{c}\text { Mayotes } \\
1 \text { año }\end{array}$ & $\begin{array}{c}\text { Menores } \\
1 \text { año }\end{array}$ \\
\hline Enero & 751 & 857 & Abril & 700 & 430 \\
\hline Fubrero & 636 & 610 & Mayo & 84 & 455 \\
\hline Marz & 660 & 50 & Junio & 1.00 & 456 \\
\hline Octubre & 1.193 & 540 & Ju'io & 1.0 & 540 \\
\hline Novbre. & 977 & ค. 69 & Agosto & 1.07 & 478 \\
\hline Dcbre. & 003 & 779 & Sepbre. & 1.116 & 566 \\
\hline Total. & 5.076 & 9.958 & Total & 5.784 & 2.925 \\
\hline
\end{tabular}

Dicho de otro modo, mientras la mortalidad estival por infecciones respiratorias agudas, en menores de 1 año, es un $57,5 \%$ de la mortalidad infantil, en los mayores de un año la misma proporción es del $46,74 \%$.

En Magallanes, donde el clima es riguroso y parejo todo el año, donde la patología respiratoria es abundante y donde la patología infecciosa digestiva es baja, las enfermedades respiratorias agudas de invierno y otoño, hacen el $\mathbf{5 5 \%}$ de las muertes infantiles anuales. Nonde baja la morbilidad digestiva estival, baja la mortalidad respiratoria estival.

O sea, la mortalidad infantil chilena por bronconeumonía, neumonía y gripe es alta, y tiene preferencia estival, contrariando la experiencia epidemiológica general y la distribución de la mortalidad del mayor de un año, por esos misinos cuadros. $y$ difiere de jo que ocurre en la provincia fría de Magallanes.

¿Cuál es la explicación de este hecho? No la conocemos. Pero mientras no tengamos una certificación médica suficiente $y$ eficiente de estos casos, tenemos derecho a suponer que la distribución estival de estas muertes está escondiendo muertes infantiles por trastornos digestivos agudos, característicos de las estaciones calurosas. El certificado de defunción estaría viciado por la ignorancia del testigo o por el registro de la enfermedad terminal.

Efectivamente, Gallo (17) sorprendido porque la bronconeumonía alcanzara el $64 \%$ de 
los diagnósticos de muertes infaritiles en Coronel, investigó su origen. Comprobó que tan elevada frecuencia estaba condicionada por el registro de las comunidades rurales donde la bronconeumonía alcanzaba al $76 \%$, que el $\mathbf{5 6} \%$ de los certificados de defunción expedidos por el médico sanitario, al que recurrían los familiares del niño inuerto cuando éste no había recibido atención médica, registraba la increíble frecuencia de $\mathbf{9 0 \%}$ para la bronconeumonía. En los pocos casos de niños en aue pudo comparar los diaznósticos del médico tratante del $S$. $O$. con el certificado de defunción del médico sanitario, el desacuerdo alcanzaba al $50 \%$.

Ia provincia de Magallanes tiene el más alto porcentaje de certificación médica de muertes en Chile, $96 \%$. Sin embargo, el $17 \%$ de los certificados no consignaban la causa, en 1947 y 1948, por no haber sido comprobada por el médico firmante, llamado después de producido el deceso.

La expresión debilidad congénita viene desde antiguo, su definición no ha sido precisada $y$, probablemente, incluye una gran cantidad de casos que diagnosticaríamos como prematuros, de usar la definición recomendada por la $\mathrm{O}$. M. S. El diagnóstico de meningitis simple no contagiosa, ha descendido al $25^{\%}$, del nivel de hace 20 años. Parece destinado a desaparecer. Su alta frecuencia anterior sugiere que haya encubierto otros diagnósticos.

Es posible que nuestras tasas de distrofia $y$ de malformaciones estén rebajadas por el registro de la causa de muerte terminal.

Las distribuciones de la Tabla $\mathrm{N}^{\ominus} 3$. corresponden a las que algunas comunidades de Estados Unidos tenían hace 40 años, aproximadamente. Ahora. Ja disminución de las infecciones digestivas estivales como consecuencia del saneamiento, y el mayor control de las enfermedades infecciosas en general, destacó en los lugares do privilegio a las causas de la mortalidad infantil cuya raíz se remonta a épocas anteriores al parto, o al parto mismo, y que son difícilmente influenciables.

Pese a los defectos anotados es obvio que más del $50 \%$ de la mortalidad infantil chilena es infecciosa, y que de ésta, más de un tercio es digestiva. La higiene absoluta de la alimentación podría erradicarla, aparte de que influiría en todos los otros rubros con- dicionados por la desnutrición de nuestros lactantes.

Sabemos cómo hacerlo; falta que lo queramos y nos organicemos para ello. No conseguiremos erradicar todos los males, pero aseguraremos la baja de nuestras tasas hacia las proximidades de los países líderes, con la misma ambiciosa velocidad que llevamos en este momento. Por otra parte, no se trata $\$ \delta$ lo de salvar vidas infantiles, sino más bien de asegurar a cada niño iguales oportunidades de un crecimiento y un desarrollo normal. Esto es una responsabilidad conjunta del clinico, del investigador, del higienista y de la sociedad.

Al médico clínico le corresponde desempeñar da más importante tarea de esta secuencia. La de diagnosticar y tratar los casos individuales, tanto desde el punto de vista curativo como preventivo, además de proveer la información clínica básica que hará posible la labor sanitaria y social que otros encauzarán a base de su cooperación.

Sin el diagnóstico y denuncio oportuno de la incidencia de determinadas enfermedades infecciosas por el médico tratante, no será nosible nunca emprender con éxito el control de una epidemia.

Sin el diagnóstico acucioso de la causa inmediata de muerte y de la causa inicial oue origina la cadena de acontecimientos que lleva a la muerte y su registro, el sanitario no podrá concertar sus recursos en aquellas que merecen un interés más intenso; sea por su elevada incidencia, sea porque conocemos técnicas eficaces para combatirlas, sea porque la fácil aplicabilidad de éstas y sus baios costos, aseguran un alto rendimiento de los dineros y esfuerzos que la comunidad distrae de otras acciones, para comprar salud. Por tanto, el médico tratante tiene una doble responsabilidad; ante el individuo $y$ ante la colectividad.

Parte de esta última debe cumplirla informando con exactitud de sus hallazgos. Durante un largo tiempo previsible, las causas de muerte serán las que definan más exactamente los riesgos sanitarios de las poblaciones; de ahí la importancia del certificado de defunción que el médico redacta con acuciosidad.

La discusión anterior demuestra gue nuestros registros son aún defectuosos. Como este fenómeno es más o menos general en el mundo entero, la sexta Revisión Decenal In- 
ternacional de Causas de Muerte, realizada en París en 1948, aprobó una nomenclatura standard para el registro de enfermedades $y$ traumatismos y causas de muerte y un Certificado de Defunción standard para uso internacional. Chile, signatario de dicho acuerdo, obtuvo que el Colegio Médico, el Servicio Nacional de Salud y las Direcciones General de Registro Civil y Estadística lo adoptaran.

Tenemos, así, un instrumento que perfeccionará en el futuro nuestro conocimiento de la mortalidad general e infantil.

La cooperación de los médicos es decisiva para que rinda los frutos que se esperan de él. Su manejo es simple, requiere poco más trabajo que el certificado antes vigente; sus instrucciones son fácilmente comprensibles. Si el médico toma conciencia de la importancia que sus informes tienen para programar los planes sanitarios se sentirá estimulado a gastar una media hora inicial en estudiar su maneio $y$ después todo será fácil.

Si hubiéramos contribuído a aclarar sólo este punto, nos sentiriamos satisfechos de haber cansado la atención de ustedes, además de anunciarles para un futuro próximo la posibilidad de discutir un programa de qué hacer y cómo hacerlo para mejorar las oportunidades sanitarias del niño chileno y llegar a ser merecedores de la reputación que ganaron para la Pediatría Chilena, nuestros predecesores.

\section{Resumen}

Se estudia la mortalidad infantil chilena desde un punto de vista epidemiológico y según diagnóstico médico.

El estudio epidemiológico evidencia que la mortalidad infantil chilena es alta comparada con los países que la registran en la O. M. S., aunque muestra constante disminución en los últimos decenios, disminución que se ha acelerado a partir de 1936. Aparece asociada a factores genéticos $y$ concepcionales como el sexo del niño, su peso de nacimiento, la edad y paridad de la madre $y$ a un conjunto de influencias externas, difíciles de separar, como las condiciones económicas, la concentración urbana, las tasas de natalidad y de legitimidad, la atención del parto en maternidad, las estaciones del año, la edad y la alimentación del niño, etc.
La mayor mortalidad estival y la relativa mayor mortalidad tardía que neonatal, șugieren que el peso de las condiciones adversas del ambiente son aún muy fuertes en nuestro país. Iaas enfermedades infecciosas causan más del $50 \%$ de las muertes infantiles. El saneamiento ambiental es aún en Chile la acción sanitaria más importante para rebajar la mortalidad infantil.

El análisis de las enfermedades que registran el mayor número de muertes infaniiles. demuestra que aún es muy elevada la certificación no profesional y que la certificación médica no es eficiente. La especial importancia que un conocimiento exacto de las causas de muertes infantiles tiene para conducir programas de higiene infantil, hace necesaria la adopción de las normas de certificación recomendadas por la Comisión Internacional que hizo la $6^{9}$ revisión $\mathrm{y}$ cuyas ventajas se están divulgando.

\section{Summary}

A study is made of infantile mortality in Chile under the epidemical point of view and according to medical diagnosis.

The epidemiological study shows that infant mortality in Chile is high compared with other countries that register it in W. H. O., although it shows constant diminution in the last decades, diminution that has accelerated since 1936. It appears associated with conceptional and genetic factors such as sex of the child, weight at birth, age and parturition of the mother and a combination of external influences, difficult to separate such as economic conditions, urban concentration, the percentage of birth and legitimity, medical attention during birth, the seasons of the year, age and nutrition of the child, etc.

The "greater Winter mortality and the relatively greater late mortality than neonatal, suggest that the weight of adverse conditions of the weather are still very strong in our country. Infectious diseases cause more than $50 \%$ of infant deaths. The bettering of sanitary standards is still in Chile the most important sanitary action to lower infant mortality.

Analysis of the diseases that register the greater number of infant deaths show that the non-professional certificate is still very high, and that the medical certificate is not 
efficient. The special importance that exact knowledge of the causes in infant deaths makes necessary the adoption of the norms of certification recommended by the International Commision which made the 6th revision and whose advantages are now being shown.

\section{$B I B L I O G R A F I A$}

1. DUBLIN, Y COLS.-"Length of life". N. Y, The Ronald Press, 1949.

2. SAND, RENE.-"Vers la Médécine Social". Paris, Ballière et fils, 1948.

3. PASSMORE, R.-"Nutrition and Health of Children in five Countrics of $\mathrm{S}$. A." Cnicef. Pub. No E-ICEF-83, 1948.

4. ROMERO Y UGARTE-" Mottalidad Infantil", Rev. Chi], de Hig. y Med. Prev. XI: 143-163, 1949.

5. MORODFR J. Y COLS.-"Mortalidad Infantil en Chile", Rev. Chil, de Hig. y Med. Prev., XIII: 8392, Dic. 1951

6. ADRIASOLA G., Y COLS.-"Atención deI recién nacido en las Maternidades de Santiago". Actas de las 2.as Jornadas Chil. de Salubridad, Santiago. 1952.

7. THE CHILDREN'S BUREAU.-F. S. A. U. S. A "The premature children, a Mannual for Phisicians",

8. CABEILO, O.-"Influencia de la Unidat Sanitaria Quinta Normal en la reducción de la morta- lidad infantil de la comuna". Rev. Chil. de Hig. y Med. Prev., VIII: 15-27, junio, 1946.

9. YERUSHALMY, J.-"Studies on Childbirth Mortality", P. H. Reports, 55: L1-95, julio, 1940.

10. LEVINE F., Y GROCCO, J.-"La población chilena", Rev. de la Fac. de Economia de Chile, v: \$168, julio, 1944.

I1. ALTENDERFER, M., CROWTHER, B.-"Relaciones entre mortalidad infantil y factores socio. económicos en áreas urbanas". P, H. Reports, 64: 331-339, 1949.

12. STEVENSON, $\mathbf{S} \rightarrow$ "The adequacy of artificial feeding in infancy". The Journal of Pediatrics, 31: 316,1947

"Comparison of Breest and artificial feedings". Journal of the Am. Dietetics Ass., 25: 752-756, 1949.

13. MARDONES, FCO.-"Alimentación del lactante sano en servicios de Protección Matemo Infantil". Reץ. Chil. de Pediatría, XXII: 415-427, 1951.

14. NICOLAI, J. F.-“Natalidad \% Mortalidad Infantil". Anales de la Universidad de Chile, XCI, No 12, pisg. 167, 1933.

15. ORTFGA, R.-"Una interpretación de las causas de mortalidad infantil en Chile". Bol. Med. Soc., IX: $456-460,1942$

16. EASTMAN, PARIS R.-"Infant Mortality in relation to morth of birth". Am. J. of. P. Health, 35 : 913-922, septiembre, 1945 .

17. GAILO, ARTURO.--"Influencia de la sección Madre y Nifio de la Caja de Seguro Obrero, sobre la Mortalidad Infantil en Coronel". Rev. Chil. de Hig. Y Med. Prev., XII: 55-66, 1950. 